\title{
Optimal Imaging Strategies for Rectal Cancer Staging and Ongoing Management
}

\author{
Svetlana Balyasnikova, MD, PhD $1,2,3,4$ \\ Gina Brown, MD, FRCR ${ }^{1,2, *}$
}

\author{
Address \\ *,1 Colorectal Imaging Group, The Royal Marsden Hospital, NHS Foundation Trust, \\ Downs Road, Sutton, Surrey, SM2 5PT, UK \\ Email: gina.brown@rmh.nhs.uk \\ ${ }^{2}$ Imperial College London, London, UK \\ ${ }^{3}$ The N.N. Blokhin Cancer Research Center, Kashirskoye Shosse 24, 15478, Mos- \\ cow, Russia \\ ${ }^{4}$ The National Scientific Center of Coloproctology, ul. Saliama Adilia 2, 123423, \\ Moscow, Russia
}

Published online: 2 June 2016

(C) The Author(s) 2016. This article is published with open access at Springerlink.com

This article is part of the Topical Collection on Lower Gastrointestinal Cancers

Keywords Rectal cancer staging · High-resolution MRI - Early and advanced rectal cancer - ERUS - EMVI • CRM - PET/ $\mathrm{CT} \cdot \mathrm{N}$ stage $\cdot$ EMVI $\cdot$ Response assessment $\cdot$ Recurrent and mucinous rectal cancer

\section{Opinion statement}

Imaging determines the optimal treatment for rectal cancer patients. High-resolution magnetic resonance imaging (MRI) overcomes many of the known limitations of previous methods. When performed in accordance with the recommended standards, MRI enables accurate staging of both early and advanced rectal cancer, accurate response assessment, the delineation of recurrent disease and planning surgical treatment in a safe and effective manner. Tumour-related high-risk features with known adverse outcomes can be preoperatively identified and treated with neoadjuvant chemoradiotherapy. Further, MRI posttreatment tumour response assessment using TRG grading system also predicts the likely survival outcomes and in the future will be used to modify treatment further by stratification into good and poor responders. There is a paucity of literature with validated outcome data concerning use of diffusion-weighted imaging and positron emission tomography (PET)/computed tomography (CT), and in the absence of any validated methods and outcome data, their use in the initial assessment and restaging after treatment is limited to research protocols. Combination MRI and CT is essential for distant spread assessment and recurrent disease, and currently PET-CT is sometimes used in the workup of patients with recurrent and metastatic disease. 


\section{Introduction}

Preoperative assessment of tumour spread has become essential for both early and locally advanced rectal cancer, response assessment to neoadjuvant chemoradiotherapy (CRT) and resectability of recurrent disease. A number of different imaging modalities have been used to assess locoregional and distant tumour spread, with magnetic resonance imaging (MRI) use predominating for rectal cancer staging in the last 10 years. Nowadays, for the majority of colorectal units, imaging assessment of the primary tumour helps to determine the surgical procedures and the necessity for preoperative treatment.

Implementation of bowel cancer screening programs has increased the rate of patient identified with early rectal cancers (ERC, defined as small early stage tumours, T1/T2 and polyps) [1] who could potentially benefit from adequately less aggressive surgical treatment; imaging is mandatory for extramural spread assessment and modern high-resolution methods can even be used to define the degree of preservation of submucosa and muscularis propria (provided that the imaging quality complies with published standards) [2]. Specific imaging features of tumour aggressiveness proven to predict outcomes such as magnetic resonance (MR)-circumferential resection margin (CRM) [3] and MR presence of extramural vascular invasion (EMVI) [4] should also be taken into consideration when treatment decisions are made in order to reduce risks of both local and distant failure in rectal cancer patients.

Imaging is becoming necessary for tumour response assessment when patients have completed neoadjuvant therapy. Although histopathology remains the so-called gold standard for evaluating tumour response within the final specimen, the opportunity to modify treatment before definitive surgery takes place is missed. Thus, preoperative identification of imaging complete responders (cr) will enable detection of patients who could potentially undergo organ-preserving treatment in the future and trials are underway to test the performance of MRI in the identification and surveillance of such patients. Conversely, preoperative detection of poor responders may lead to further consolidation therapy in order to reduce risks of local and distant failure. Thus, the post-treatment assessment of response using high-resolution MRI may in the future become a crucial step in the management of patients with advanced rectal cancer (TRIGGER: EudraCT Number 2015-003009-40).

Patients with locally recurrent rectal cancer form a challenging group for cure due to limited treatment option as CRT and surgery have usually been offered previously. The importance of accurate imaging has been recognised for these patients; a risk stratification staging system is based on number and location of pelvic compartments involved as identified on highresolution MRI. This method of assessment has been shown to predict clinical and survival outcomes $[5,6 \bullet]$.

Imaging modalities involved in the local and distant staging of rectal cancer are the following: endorectal ultrasound (ERUS), MRI, computed tomography (CT) and positron emission tomography (PET)/CT (a summary of different methods performance is listed in a Table 1). Both ERUS and MRI are commonly recommended for ERC staging, and there is also strong agreement that MRI has become a required standard for evaluating locally advanced disease particularly those patients with potential CRM involvement. According to NCCN (North America) [7]/NICE (UK) [8] guidelines, contrast-enhanced CT of the chest, abdomen and pelvic should be offered to rectal cancer patients in order to estimate distant spread of the disease [8]. Excessive cost and time of a PET/CT scan limits its role to second choice modality when CT or MRI results are ambiguous in assessment of distant tumour spread. There are many discussions regarding the use of PET/CT for evaluating response to treatment; however, no consensus has been found in performing PET/CT in this context. Nevertheless, a combination of different modalities, including $\mathrm{PET} / \mathrm{CT}$, is almost always required in imaging of recurrent disease $[9,10]$.

\section{ERUS}

ERUS with the rigid probe has a number of imaging limitations including inability to stage high, bulky and structuring tumours [11]. Use of either a 7-10$\mathrm{MHz}$ or flexible rigid ERUS ultrasound probe results in a small field of view (FOV) that prevents assessment of tumour relationship to the potential 


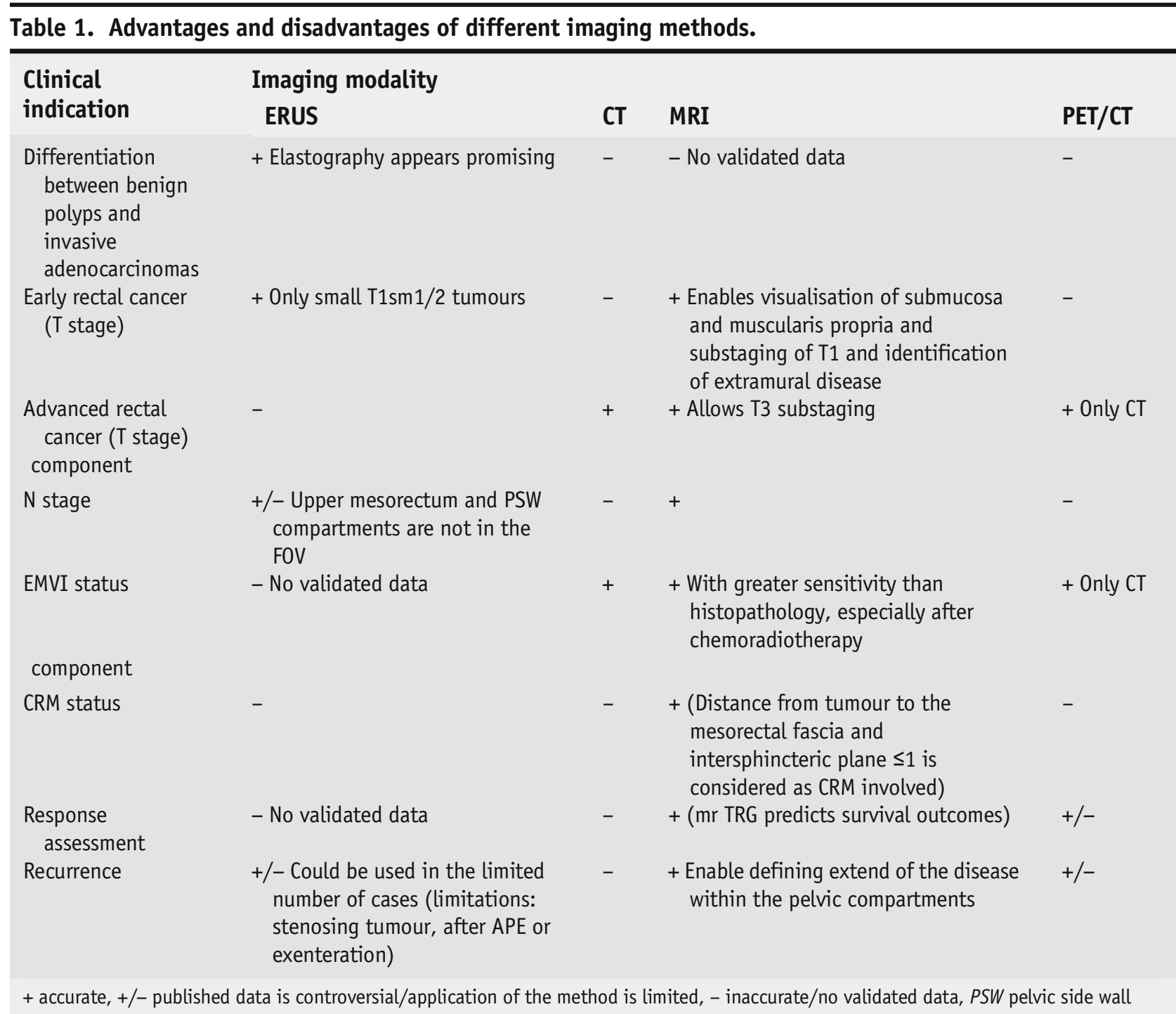

circumferential margin (CRM). Inability to distinguish peritumoral fibrosis from tumour spread limits use of ERUS in performing both primary and postCRT assessment of rectal cancer [12]. The crucial limitation of ERUS assessment techniques is the limited views of the whole mesorectum and pelvis to exclude tumour deposits, discontinuous vascular invasion and mesorectal fascia involvement by tumour. Thus, disease beyond the immediate vicinity of the primary tumour is impossible to evaluate using ERUS techniques and will inevitably lead to understaging. According to the published meta-analysis, ERUS does not perform any differently to either CT or MRI. However, this analysis limited assessment to $\mathrm{T}$ and $\mathrm{N}$ staging only and did not take into account the important prognostic variables that should also be assessed by imaging, namely depth of extramural spread in millimetres, relationship of tumour to the mesorectal fascia and extramural vascular invasion [13]. It has been suggested that the published literature overestimates the performance of ERUS in staging rectal cancer with median T stage accuracy of $69 \%$ and $\mathrm{N}$ stage 
of accuracy $65 \%$ for studies of $>300$ subjects-again there is no published data on the ability of ultrasound to stratify prognostic groups [14]. Despite many expectations, ERUS has not proven to be effective in staging ERC, as preoperative staging by these methods does not influence local recurrence or tumourspecific survival [15]. The UK transanal excisional microsurgery (TEMS) registry group reported that ERUS inaccurately staged rectal cancer in $44.8 \%$ out of 165 patients who underwent TEMS for ERC, and no significant difference was found in the depth of TEM excision or R1 rate between the patients who underwent ERUS before TEM and those who did not $(p=0.73)$ [16]. As an assessment tool for potentially significant polyps, ERUS with an elastography algorithm could potentially be useful for differentiating malignant transformation within rectal adenomas; a study by JER Waage et al. showed that strain elastography could assess tissue hardness which enabled distinction between benign adenomas vs invasive adenocarcinomas with $0.86(0.66-0.96)$ specificity and $0.94(0.88-0.97)$ accuracy when compared to final histopathology [17].

The overall multidetector CT accuracy of T staging is around $86 \%$ [18]; however, its sensitivity to predict relationship of the tumour to CRM is less than $50 \%$ [19], making the method unreliable for clinical practice. Invasion of the adjacent structures may be difficult to assess due to lack of fat plane between the tumour, mesorectal fascia and adjacent structures anteriorly or in the lower rectum.

For lymph node assessment, size is most commonly used CT criterion for differentiating benign from malignant lymph nodes; however, Bipat et al. metaanalysis showed that CT had $55 \%$ sensitivity and $74 \%$ specificity for predicting lymph node involvement in rectal cancer patients [13].

Nevertheless, published data suggests that such risk factors of tumour spread as mucinous content and presence of EMVI could be identified on CT scans. Heterogeneous enhancement and presence of areas of hypoattenuation are more frequently identified within the mucinous tumour vs non-mucinous [20]. It has been shown that CT allows visualisation of EMVI characterised by nodularity and expansion of perirectal vessels [21], and in colon cancers, it can be used to stratify good vs poor prognosis using depth of extramural spread of $>5 \mathrm{~mm}$ as a definition for poor-risk tumours $[22,23]$.

MRI has become the optimal modality for the local staging of primary and recurrent rectal cancer. There are several advantages over alternative techniques: it enables to stratify tumours depending on the presence of several high-risk features that are not limited to T and N stage (CRM and EMVI status, depth of extramural invasion, presence of discontinuous extramural vascular spread/ deposits, presence of mucin and grade of tumour regression to preoperative treatment). These have all been proven to influence disease-free and overall survival rates in prospective multicentre studies. In recurrent rectal cancer, MRI allows the delineation of tumour extent within the pelvic compartments, can assess the pattern and mode of local recurrence and predict resectability of the disease. 
Specific imaging parameters should be followed to achieve optimal results of the MRI technique. Good quality high-resolution scan can be achieved if the following technique criteria are satisfied (Table 2):

- Small FOV (not more than $160 \mathrm{~mm}$ ) coronal, sagittal and axial slices

- Slice thickness (no more than $3 \mathrm{~mm}$ and voxel size less than 1.5)

- Correct scan plane alignment (perpendicular to the rectal wall at the level of tumour advancing edge) $0 x(\wedge$ list $)$

The voxel and FOV size increase will cause loss of resolution, so that assessment of tumour morphological characteristics becomes challenging. Incorrect imaging plane incline usually leads to tumour overstaging.

Intravenous contrast to assess primary rectal cancer or recurrence is not recommended as there is no evidence that its use improves accuracy or gives additional prognostic staging information.

\section{Primary Tumour Staging}

\section{T Stage}

On high-resolution MRI scans, rectal wall anatomy is clearly depicted so depth of tumour invasion can be correctly assessed. Not only is extension into the mesorectum can be evaluated but also tumour spread within the layers of the bowel wall. Therefore, MRI enables to identify patients with early rectal tumours eligible for organ-sparing surgical treatment (local excision) and those with high-risk tumours, when preoperative treatment is mandatory.

\section{Early Rectal Cancer Staging}

All rectal cancers apart from mucinous tumours demonstrate intermediate signal intensity (SI), whereas the submucosal layer shows hyperintensity and the muscularis propria (MP) low-intensity MR signal. The presence of a hyperintense stripe between the tumour and muscularis at the level of tumour

Table 2. Optimal MRI protocol for achieving high-resolution scans.

\begin{tabular}{lllll}
\hline Sequence & Sag TSE T2 & Axial TSE T2 & Axial TSE T2 high-resolution & Cor TSE T2 \\
TR & 3961 & 4018 & 5362 & 5362 \\
TE & 125 & 80 & 100 & 100 \\
TSE factor & 23 & 20 & 16 & 16 \\
FOV/RFOV & $250 / 100 \%$ & $300 / 100 \%$ & $160 / 90 \%$ & $160 / 90 \%$ \\
Slice thickness/gap & $3 / 0.4$ & $5 / 1$ & $3 / 0.3$ & $3 / 0.3$ \\
NSA & 4 & 2 & 6 & 6 \\
Matrix & $320 / 512$ & $256 / 512$ & $256 / 256$ & $256 / 256$ \\
Sat bands & Ant/Sup & None & None & None \\
Acquisition time & 6.00 & 3.28 & 7.35 & 7.35 \\
$T R$ repetition time, $T E$ echo time, TSE turbo spin echo, FOV field of view, RFOV reduced field of view
\end{tabular}


advancing border (not the rolled edges) should be interpreted as full or partial preservation of submucosa and indicates a likely T1sm1/2 at most; on the contrary, absence of preserved submucosa but unaffected MP suggests T1sm3/ T2 (with partial invasion of the muscularis) - from the clinical point of view, there is no need in differentiating these two stages (sm3/T2); if no macroscopic invasion of the mesorectal fat is present and no low signal intensity line identified between intermediate SI tumour and hyperintense mesorectal, tumour should be staged as T2 (with full thickness of MP invasion)/T3a $(<1 \mathrm{~mm}$ tumour spread beyond the muscularis propria) - prognosis and outcomes of patients with these $\mathrm{T}$ stages are no different, so their distinction is unnecessary.

\section{Advanced Rectal Cancer Staging}

Macroscopic spread of tumour beyond the MP associated with worsening prognosis. Tumour spread $<5 \mathrm{~mm}$ is associated with identical prognosis as T2 tumours provided that there is no CRM involvement by tumour. More advanced penetration into the mesorectum (T3c-d disease) is associated with increasing rates of disease recurrence [24, 25]. Subclassification of T3 stage based on depth of tumour spread into the mesorectum should be used, while MRI staging is performed as MRI has been proven to be accurate in substaging T3 tumours with measured equivalence to histopathology [26].

\section{Circumferential Resection Margin}

The outermost boundary of the mesorectum is defined by the mesorectal fascia (MRF), which acts as a barrier to tumour spread and demarcates the CRM during surgical excision. MRI has been proven to accurately identify potential distance from tumour to CRM within $1 \mathrm{~mm}$, which is recognised by pathologist as a clear margin $[2,27-29]$. Results of the prospective MERCURY Study showed that specificity of MRI for predicting PCRM is $92 \%$ [30].

According to the TNM classification system, tumours invading the mesorectal fascia are not T4-therefore, the predicted MRI CRM status must always be reported in addition to the TNM. A measured distance of $1 \mathrm{~mm}$ or less from the primary tumour, tumour/vascular deposit, or invaded extramural vessels to the MRF is one of the major prognostic factors for local recurrence, poor disease-free survival and poor overall survival and should always be stated in the MRI reports.

\section{N Stage}

Nodal staging is of questionable importance as a predictor for local recurrence in patients receiving radical surgery for primary tumours; however, it still should be assessed if local excision is considered. Some authors continue to suggest using size criteria when determining the nature of mesorectal nodes; however, there is no rational basis for this and all published pathology data indicates that there is no relationship between the size of a lymph node and its likelihood of malignancy [31]. In a histological survey of over 12,000 lymph nodes from rectal cancer, specimens showed a considerable size overlap between normal, reactive and malignant nodes [31]. A prospective study where nodes from in vivo MRI, MRI specimens and pathology specimens were 
matched showed that there was no useful size cutoff for predicting nodal status [32]. Rather than size, it is the morphologic MR features such as presence of heterogeneous MR signal and irregular borders of lymph node capsular that are more reliable in differentiating nature of changes within nodes, and this has been prospectively validated in several studies $[32,33]$.

\section{EMVI}

MRI EMVI defined as tumour signal within the vasculature outside the muscularis propria has been proven to correlate with histopathology findings [4] and survival outcomes [34]. On high-resolution MRI, EMVI is demonstrated as expansion of veins due to tumour signal present within them. It is an independent risk factor for metastatic disease, and its presence in low rectal cancers is also associated with a risk of CRM involvement and consequent local recurrence $[35,36 \bullet \bullet$.

Assessment of tumour resectability is one of the crucial things in recurrent rectal cancer patients. Offering the best soft tissue contrast compared to the rest of the available imaging modalities MRI enables evaluating relationship of recurrent tumour to important landmarks-pelvic viscera, peritoneum, bones, nerves, muscles, fasciae and ligaments. It has been highlighted that accurate delineation of disease extent in relation to pelvic compartments is essential to plan the most appropriate treatment for this group of patients $[6 \bullet]$.

After preoperative CRT, up to $30 \%$ of patients will develop complete pathological response defined as no tumour identified on the final histology examinations. Modified Mandard grading system of tumour response was tested in MERCURY study and has been proven to be a reliable tool for assessing tumour

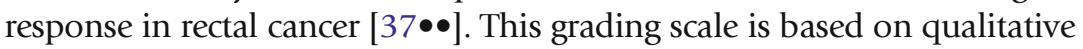
assessment of intermediate signal vs low signal (the latter considered as fibrosis and the former as residual disease) within the treated tumour. MR TRG 1-2 can be described as low signal intensity scar or low density fibrosis with no evident macroscopic intermediate signal intensity within it, mrTRG3 dominant fibrosis outgrowing the tumour mass and mrTRG 4-5 predominantly intermediate signal intensity with minimal or no signs of fibrosis. MERCURY experience showed that patients with complete and near complete response (mrTRG 1-3) have better prognosis, disease-free and overall survival compared to poor responders [38].

Some studies suggest using DW-MRI in order to increase accuracy of tumour response assessment and specificity for selecting patients eligible for deferral of surgery trial, proposing that patients showing residual diffusion restriction on high $b$ value DW images should not be offered watch and wait policy [39, 40]. However, there is no published data proving the efficacy of applying DWI in response assessment. Thus far, no publication has proven any added value of DWI compared against mrTRG, ypT, ymrT, in assessing response, overall and disease-free survival rates. A recent analysis has shown that only $32 \%$ of patients with imaging complete response (mrTRG1-3) would have been included in the protocol if diffusion-weighted criteria had been applied, yet no 
difference in the rate of tumour regrowth was identified amongst the 18 patients from DWI group (33.3\%) compared to 56 patients in the mrTRG 1-3 group $(37 \%)(p=0.82)$ [41]. The data indicates that DW-MRI not only underestimates patients with sustained complete response and does not improve specificity compared with mrTRG.

\section{PET/CT}

The role of an imaging modality that provides a combination of functional information (18F-FDG-PET) and morphologic information (CT) has not yet been defined for rectal cancer staging. This method is not recommended in the international standards of rectal cancer patients' management or guidelines for local spread assessment [7]. It has been shown that PET/CT detects colonic abnormalities larger than $13 \mathrm{~mm}$ in diameter with $90 \%$ accuracy [42]; however, the specificity for differentiating hyperplastic benign polyps from primary colorectal cancers is reported to be $43 \%$ and thus the application of this technique is uncertain [43].

The high cost and lower availability of PET are not the only disadvantages of the method. It has been shown to be inaccurate in differentiating changes within the mesorectal and pelvic lymph nodes $[43,44]$, it is also known to be insensitive for assessing mucinous tumours and there is no data suggesting that it could improve patient selection with complete response [45-47]. It is known that up to $25 \%$ of 18 F-FDG-uptake occurs in metabolically active but nonmalignant tissues, such as granulation or inflammation [48, 49]. There are several studies where response monitoring was performed and compared with morphological response, and it has been demonstrated that the reduction in SUVs was significantly greater in (histopathologically confirmed) responders than in nonresponders $[50,51]$; however, there is no consensus on the SUV reduction rates needed to predict complete response.

A meta-analysis published by Huebner et al. included 11 studies and 577 patients showed that FDG-PET had $97 \%$ sensitivity and $76 \%$ for detecting recurrent colorectal cancer [52]. However, Suga and colleagues reported that prevalence of PET-positive cases in rectal cancer patients was higher with an increase in carcinoembryonic antigen (CEA) levels ( $41 \%$ PET positivity for CEA level of 5-10 vs $83 \%$ with CEA level $>50$ ) [53]. Therefore, a rising CEA level, equivocal findings on other imaging, should be considered an indication for

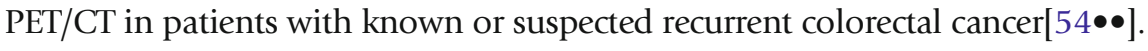

\section{Compliance with Ethical Standards}

\section{Conflict of Interest}

Svetlana Balyasnikova and Gina Brown declare that they have no conflict of interest.

\section{Human and Animal Rights and Informed Consent}

This article does not contain any studies with human or animal subjects performed by any of the authors. 
Open Access This article is distributed under the terms of the Creative Commons Attribution 4.0 International License (http://creativecommons.org/licenses/by/4.0/), which permits unrestricted use, distribution, and reproduction in any medium, provided you give appropriate credit to the original author(s) and the source, provide a link to the Creative Commons license, and indicate if changes were made.

\section{References and Recommended Reading}

Papers of particular interest, published recently, have been highlighted as:

- Of importance

$\bullet \quad$ Of major importance

1. Logan RF et al. Outcomes of the Bowel Cancer Screening Programme (BCSP) in England after the first 1 million tests. Gut. 2012;61(10):1439-46.

2. Brown G et al. Preoperative assessment of prognostic factors in rectal cancer using high-resolution magnetic resonance imaging. Br J Surg. 2003;90(3):355-64.

3. Taylor FG et al. Preoperative magnetic resonance imaging assessment of circumferential resection margin predicts disease-free survival and local recurrence: 5year follow-up results of the MERCURY study. J Clin Oncol. 2014;32(1):34-43.

4. Smith NJ et al. Prognostic significance of magnetic resonance imaging-detected extramural vascular invasion in rectal cancer. Br J Surg. 2008;95(2):229-36.

5. Moore HG et al. Colorectal cancer pelvic recurrences: determinants of resectability. Dis Colon Rectum. 2004;47(10):1599-606.

6. Georgiou PA et al. Diagnostic accuracy and value of magnetic resonance imaging (MRI) in planning exenterative pelvic surgery for advanced colorectal cancer. Eur J Cancer. 2013;49(1):72-81.

7. Network, N.C.C. NCCN clinical practice guidelines in oncology: rectal cancer, version 1.2016. J Natl Compr cancer Netw. 2015.

8. Poston GJ et al. Diagnosis and management of colorectal cancer: summary of NICE guidance. BMJ. 2011;343:d6751.

9. Schaefer O, Langer M. Detection of recurrent rectal cancer with CT, MRI and PET/CT. Eur Radiol. 2007;17(8):2044-54.

10. Colosio A et al. Local colorectal cancer recurrence: pelvic MRI evaluation. Abdom Imaging. 2013;38(1):72-81.

11. Akasu T et al. Limitations and pitfalls of transrectal ultrasonography for staging of rectal cancer. Dis Colon Rectum. 1997;40(10 Suppl):S10-5.

12. Maier AG et al. Peritumoral tissue reaction at transrectal US as a possible cause of overstaging in rectal cancer: histopathologic correlation. Radiology. 1997;203(3):785-9.

13. Bipat $S$ et al. Rectal cancer: local staging and assessment of lymph node involvement with endoluminal US, CT, and MR imaging-a meta-analysis. Radiology. 2004;232(3):773-83.

14. Harewood GC. Assessment of publication bias in the reporting of EUS performance in staging rectal cancer. Am J Gastroenterol. 2005;100(4):808-16.

15. Garcia-Aguilar J et al. Local excision of rectal cancer without adjuvant therapy: a word of caution. Ann Surg. 2000;231(3):345-51.

16. Ashraf $S$ et al. A critical appraisal of endorectal ultrasound and transanal endoscopic microsurgery and decision-making in early rectal cancer. Color Dis. 2012;14(7):821-6.

17. Waage JE et al. Endorectal ultrasonography, strain elastography and MRI differentiation of rectal adenomas and adenocarcinomas. Color Dis.

2015;17(2):124-31.

18. Sinha R et al. Diagnostic value of multidetector row CT in rectal cancer staging: comparison of multiplanar and axial images with histopathology. Clin Radiol. 2006;61(11):924-31.

19. Wolberink SV et al. Conventional CT for the prediction of an involved circumferential resection margin in primary rectal cancer. Dig Dis. 2007;25(1):80-5.

20. Ko EY et al. CT differentiation of mucinous and nonmucinous colorectal carcinoma. AJR Am J Roentgenol. 2007;188(3):785-91.

21. Burton $\mathrm{S}$ et al. Accuracy of CT prediction of poor prognostic features in colonic cancer. Br J Radiol. 2008;81(961):10-9.

22. Smith NJ et al. Preoperative computed tomography staging of nonmetastatic colon cancer predicts outcome: implications for clinical trials. Br J Cancer. 2007;96(7):1030-6.

23. Foxtrot Collaborative G. Feasibility of preoperative chemotherapy for locally advanced, operable colon cancer: the pilot phase of a randomised controlled trial. Lancet Oncol. 2012;13(11):1152-60.

24. Merkel S et al. The prognostic inhomogeneity in pT3 rectal carcinomas. Int J Color Dis. 2001;16(5):298304.

25. Steel MC et al. Extent of mesorectal invasion is a prognostic indicator in T3 rectal carcinoma. ANZ J Surg. 2002;72(7):483-7.

26. Group MS. Extramural depth of tumor invasion at thin-section MR in patients with rectal cancer: results of the MERCURY study. Radiology. 2007;243(1):132-9. 
27. Brown G. Thin section MRI in multidisciplinary preoperative decision making for patients with rectal cancer. Br J Radiol. 2005;78(Spec No 2):S117-27.

28. Taylor FG et al. One millimetre is the safe cut-off for magnetic resonance imaging prediction of surgical margin status in rectal cancer. Br J Surg. 2011;98(6):872-9.

29. Blomqvist L et al. Rectal adenocarcinoma: assessment of tumour involvement of the lateral resection margin by MRI of resected specimen. Br J Radiol. 1999;72(853):18-23.

30. Group MS. Diagnostic accuracy of preoperative magnetic resonance imaging in predicting curative resection of rectal cancer: prospective observational study. BMJ. 2006;333(7572):779.

31. Dworak O. Morphology of lymph nodes in the resected rectum of patients with rectal carcinoma. Pathol Res Pract. 1991;187(8):1020-4.

32. Brown $\mathrm{G}$ et al. Morphologic predictors of lymph node status in rectal cancer with use of high-spatialresolution MR imaging with histopathologic comparison. Radiology. 2003;227(2):371-7.

33. Koh DM et al. Evaluating mesorectal lymph nodes in rectal cancer before and after neoadjuvant chemoradiation using thin-section T2-weighted magnetic resonance imaging. Int J Radiat Oncol Biol Phys. 2008;71(2):456-61.

34. Chand $\mathrm{M}$ et al. The prognostic significance of postchemoradiotherapy high-resolution MRI and histopathology detected extramural venous invasion in rectal cancer. Ann Surg. 2015;261(3):473-9.

35. Chand $\mathrm{M}$ et al. EMVI-positive stage II rectal cancer has similar clinical outcomes as stage III disease following pre-operative chemoradiotherapy. Ann Oncol. 2014;25(4):858-63.

$36 . \bullet$ Battersby NJ et al. Prospective validation of a low rectal cancer magnetic resonance imaging staging system and development of a local recurrence risk stratification model: the MERCURY II study. Ann Surg. 2015;263(4):751-60.

Prospective study showing the importance of MRI staging, significance of mrEMVI and mrCRM status in the low rectal cancer patients.

37. Patel UB et al. Magnetic resonance imaging-detected tumor response for locally advanced rectal cancer predicts survival outcomes: MERCURY experience. J Clin Oncol. 2011;29(28):3753-60.

38. Smith JJ et al. Organ Preservation in Rectal Adenocarcinoma: a phase II randomized controlled trial evaluating 3-year disease-free survival in patients with locally advanced rectal cancer treated with chemoradiation plus induction or consolidation chemotherapy, and total mesorectal excision or nonoperative management. BMC Cancer. 2015;15:767.

39. Maas $\mathrm{M}$ et al. Assessment of clinical complete response after chemoradiation for rectal cancer with digital rectal examination, endoscopy, and MRI: selection for organsaving treatment. Ann Surg Oncol. 2015;22(12):387380.

40. S. Balyasnikova, J.B., M. Siddiqui, B. Heald, D. Tait, G. Brown, MRI enrolment criteria for deferral of surgery. Colorectal Disease. Special Issue: Abstracts of the ESCP 9th Scientific and Annual Meeting, 24-26 September, 2014: Barcelona, Spain, 2014: p. 59.

41. Yasuda $\mathrm{S}$ et al. 18F-FDG PET detection of colonic adenomas. J Nucl Med. 2001;42(7):989-92.

42. Abdel-Nabi H et al. Staging of primary colorectal carcinomas with fluorine-18 fluorodeoxyglucose wholebody PET: correlation with histopathologic and CT findings. Radiology. 1998;206(3):755-60.

43. Mukai $\mathrm{M}$ et al. Preoperative evaluation by whole-body 18F-fluorodeoxyglucose positron emission tomography in patients with primary colorectal cancer. Oncol Rep. 2000;7(1):85-7.

44. Martoni AA et al. Prospective study on the FDG-PET/CT predictive and prognostic values in patients treated with neoadjuvant chemoradiation therapy and radical surgery for locally advanced rectal cancer. Ann Oncol. 2011;22(3):650-6.

45. Palma P et al. The value of metabolic imaging to predict tumour response after chemoradiation in locally advanced rectal cancer. Radiat Oncol. 2010;5:119.

46. Berger KL et al. FDG PET evaluation of mucinous neoplasms: correlation of FDG uptake with histopathologic features. AJR Am J Roentgenol. 2000;174(4):1005-8.

47. Kubota R et al. Intratumoral distribution of fluorine18 -fluorodeoxyglucose in vivo: high accumulation in macrophages and granulation tissues studied by microautoradiography. J Nucl Med. 1992;33(11):1972-80.

48. Larson SM. Cancer or inflammation? A Holy Grail for nuclear medicine. J Nucl Med. 1994;35(10):1653-5.

49. Guillem JG et al. Sequential preoperative fluorodeoxyglucose-positron emission tomography assessment of response to preoperative chemoradiation: a means for determining longterm outcomes of rectal cancer. J Am Coll Surg. 2004;199(1):1-7.

50. Amthauer $\mathrm{H}$ et al. Response prediction by FDGPET after neoadjuvant radiochemotherapy and combined regional hyperthermia of rectal cancer: correlation with endorectal ultrasound and histopathology. Eur J Nucl Med Mol Imaging. 2004;31(6):811-9.

51. Huebner RH et al. A meta-analysis of the literature for whole-body FDG PET detection of recurrent colorectal cancer. J Nucl Med. 2000;41(7):1177-89.

52. Suga $\mathrm{T}$ et al. Prevalence of positive FDG-PET findings in patients with high CEA levels. Ann Nucl Med. 2010;24(6):433-9. 
53. Vitola J, Delbeke D. Positron emission tomography for evaluation of colorectal carcinoma. Semin Roentgenol. 2002;37(2):118-28.

$54 . \bullet$ Patel UB et al. MRI after treatment of locally advanced rectal cancer: how to report tumor response-the
MERCURY experience. AJR Am J Roentgenol. 2012;199(4):W486-95.

Manuscript describes how to assess tumour response on preoperative MRI and how to report mrTRG, which has been proven to predict survival outcomes. 